\title{
Synthesis and Anti-Tumor Activities of Fluoride-Containing Gossypol Derivatives Compounds
}

\author{
Liying Zeng1, Yijian Deng1, Lidong Weng1, Zhijun Yang², Huoji Chen"1, Qiang Liu1 \\ ${ }^{1}$ School of Traditional Chinese Medicine, Southern Medical University, Guangzhou, China; ${ }^{2}$ School of \\ Chinese Medicine, Hong Kong Baptist University, Hong Kong, China
}

Correspondence to: Huoji Chen, Chenhuoji2005@126.com; Qiang Liu, Gzlq2002@163.com

Keywords: Gossypol Derivatives, Fluoride-Containing, Synthesis, Anti-Tumor Activity

Received: August 30, $2017 \quad$ Accepted: September 16, $2017 \quad$ Published: September 19, 2017

Copyright (c) 2017 by authors and Scientific Research Publishing Inc.

This work is licensed under the Creative Commons Attribution International License (CC BY 4.0).

http://creativecommons.org/licenses/by/4.0/

(c) $\underset{\mathrm{EY}}{ } \mathrm{i}$ Open Access

\section{ABSTRACT}

We report herein the design and synthesis of a series of novel fluoride-containing gossypol derivatives by the condensation reaction of gossypol with fluoride-containing aromatic amine. These fluoride-containing gossypol derivatives were characterized by IR, ${ }^{1} \mathrm{H}-\mathrm{NMR}$ and high resolution mass spectral data, then screened as antitumor agents against three human cancer cell lines (HeLa, A-549 and BGC-823) and a normal cell line (VEC) in vitro by using MTT cell proliferation assays. Results revealed that compounds $3 \mathrm{a}, 3 \mathrm{c}$ and $3 \mathrm{f}$ exhibited superior anticancer activity against $\mathrm{HeLa}$, compounds $3 b, 3 c, 3 e$ and $3 \mathrm{f}$ exhibited superior anticancer activity against A-549, compounds $3 \mathrm{~b}, 3 \mathrm{c}$ and $3 \mathrm{f}$ exhibited superior anticancer activity against BGC-823 compared to gossypol. In particular, fluorine substituent at the para positions of the phenyl ring showed remarkable inhibitory effects on HeLa (3c: $\mathrm{IC}_{50}$ $\left.=14.2 \mu \mathrm{M}, 3 \mathrm{f}: \mathrm{IC}_{50}=8.34 \mu \mathrm{M}\right), \mathrm{A}-549\left(3 \mathrm{c}: \mathrm{IC}_{50}=6.32 \mu \mathrm{M}, 3 \mathrm{f}: \mathrm{IC}_{50}=9.76 \mu \mathrm{M}\right)$ and BGC-823 cells $\left(3 \mathrm{c}: \mathrm{IC}_{50}=8.62 \mu \mathrm{M}, 3 \mathrm{f}: \mathrm{IC}_{50}=4.36 \mu \mathrm{M}\right)$. Furthermore, all the compounds $3 \mathrm{a}-3 \mathrm{f}$ exhibited lessened cytotoxicity against VEC compared to gossypol.

\section{INTRODUCTION}

Gossypol, the polyphenolic constituent isolated from cottonseeds, has been used as a male antifertility drug for a long time, and has been demonstrated to exhibit excellent anti-tumor activity towards multiple cancer types [1]. Up to now, gossypol has been showed to exhibit anti-tumor activities towards a wide range of tumors, such as Ehrlich ascites tumor cells [2], SW-13 adrenocortical carcinoma cells [3], hormone-dependent and hormone-independent breast carcinoma [4, 5], colon carcinoma cell line HT-29 and LoVo [6], human pancreatic cancer cell line [7], prostate cancer cell lines [8], head and neck cancer cells $[9,10]$. In addition, many synthesized gossypol derivatives and analogues possess disease-inhibiting activities [11], as anti-parasitic [12,13], anti-malarial [14-16], anti-HIV [17-19] and anticancer [20-23]. Derivatives such as gossypol Schiff bases prepared by modifying gossypol's aldehyde groups were supposed to 
reduce its host toxicity (Figure 1) while retaining or enhancing its therapeutic effects [24].

Fluorine substituents have become a widespread and important drug component. Organofluorine affects nearly all physical and adsorption, distribution, metabolism, and excretion properties of a lead compound. Its inductive effects are relatively well understood and enhancing bioavailability [25-27]. Top-selling fluorinated pharmaceuticals include the antidepressant fluoxetine (Prozac), the cholesterol-lowering drug atorvastatin (Lipitor), and the antibacterial ciprofloxacin (Ciprobay) (Figure 2) [28].

The aforementioned findings stimulated our interest in designing and synthesizing a series of fluoride-containing gossypol Schiff base derivatives (Figure 3) which were anticipated to be a much higher anti-tumor activity yet lower systemic toxicity than gossypol. The activity of the target compounds were evaluated by three human cancer cell lines (HeLa, A-549 and BGC-823) and a normal cell line (VEC) in vitro by using MTT cell proliferation assays. To the best of our knowledge, the biological evaluation of fluoride-containing gossypol derivatives for in vitro anti-tumor activity is not reported [11].

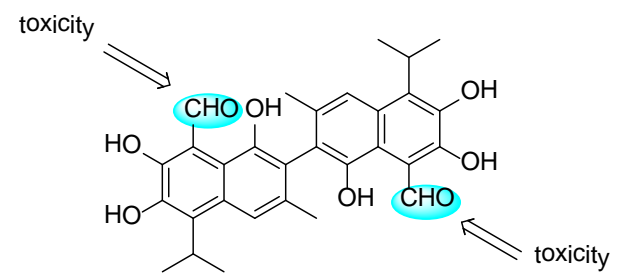

Figure 1. Chemical structure of gossypol (The highlighted aldehyde groups were supposed to be toxicity).

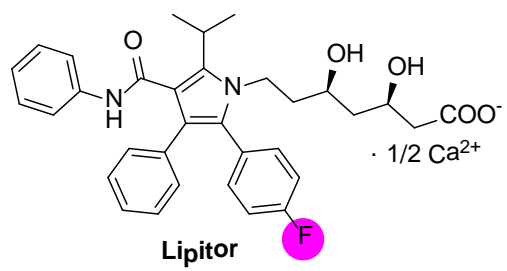<smiles>FC(F)(F)c1ccc(OC(CCNCCl)c2ccccc2)cc1</smiles>

Prozac<smiles>O=C(O)c1cn(C2CC2)c2cc(N3CCNCC3)c([18F])cc2c1=O</smiles>

Ciprobay

Figure 2. Major fluorinated drugs.<smiles>Cc1cc2c(C(C)C)c(O)c(O)c(C=O)c2c(O)c1-c1c(C)cc2c(C(C)C)c(O)c(O)c(C=O)c2c1O</smiles><smiles>C=CC=CCCc1c(O)c(C(C)C)c(O)c2c(O)c(-c3c(C)cc4c(C(C)C)c(O)c(O)c(CCCC)c4c3O)c(C)cc12</smiles><smiles>C1C[Se]2CC[As]12</smiles>

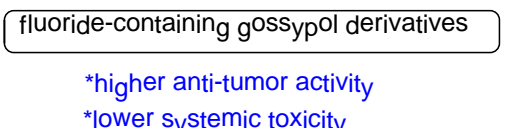

Figure 3. Designing of fluoride-containing gossypol derivatives. 


\section{RESULTS AND DISCUSSION}

\subsection{Synthesis of Gossypol Derivatives}

Fluoride-containing gossypol derivatives $3 \mathrm{a}-3 \mathrm{f}$ were prepared by the condensation reaction of gossypol1 with fluoride-containing aromatic amine $2 \mathrm{a}-2 \mathrm{f}$ (Scheme 1 ).

Reaction conditions: For preparation of fluoride-containing derivatives 3 . The gossypol (0.001 mol) dissolved in an excess of methanol $(40 \mathrm{ml})$ was mixed with 0.004 mol suitable compound as shown in Table 1 and the stirrer was added later. Next, put the reactor into the heat collection type constant temperature heating magnetic stirrer with $65^{\circ} \mathrm{C}$, the mixture was heated and refluxed for 4 hours to precipitate the yellow solid which was recovered by filtration and washed with petroleum ether-ethyl acetate (16:1). Then, the precipitate was purified by recrystallization from petroleum ether-ethyl acetate; For Compound 3, the structure can be interpreted by ${ }^{1} \mathrm{H}$ NMR (Figure 4) [22].

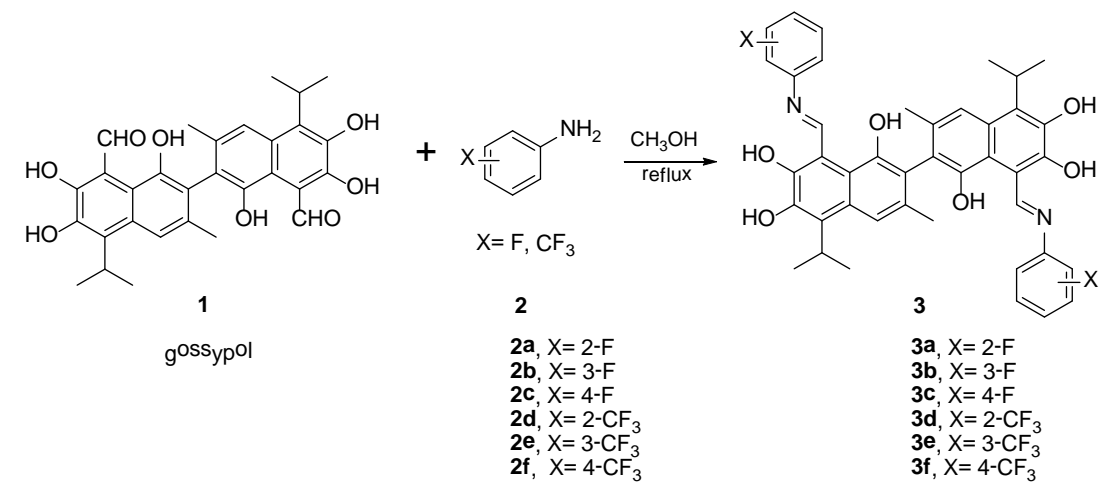

Scheme 1. Synthesis of fluoride-containing gossypol derivatives $3 a-3 f$.
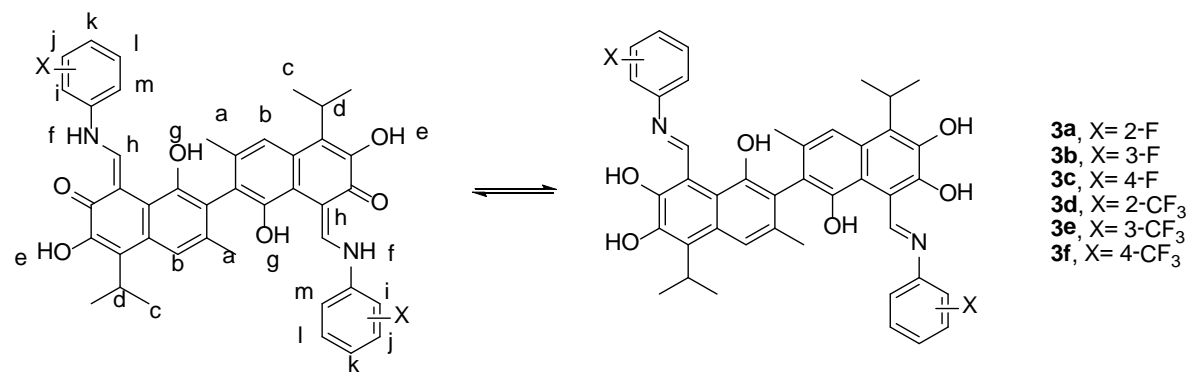

Figure 4. Attribution of proton NMR signals.

Table 1. The suitable compounds required for the preparation of fluoro-gossypol derivatives.

\begin{tabular}{cc}
\hline fluoride-containing gossypol derivatives & Compounds 2 \\
\hline $3 \mathrm{a}$ & 2 -fluoroaniline \\
$3 \mathrm{~b}$ & 3 -fluoroaniline \\
$3 \mathrm{c}$ & 4 -fluoroaniline \\
$3 \mathrm{~d}$ & 2-trifluoromethylaniline \\
$3 \mathrm{e}$ & 3-trifluoromethylaniline \\
$3 \mathrm{f}$ & 4-trifluoromethylaniline \\
\hline
\end{tabular}


8,8'-bis((E)-(2-fluorophenylimino)methyl)-5,5'-diisopropyl-3,3'-dimethyl-2,2'-binaphthyl-1,1',6,6',7,7' -hexaol (3a): yellow solid, Yield: $0.61 \mathrm{~g}$, yield: $75 \%$; mp, $255^{\circ} \mathrm{C}-257^{\circ} \mathrm{C}$; IR $\left(\mathrm{KBr}, \mathrm{cm}^{-1}\right)$ : 3352, 3102, 1580 , $1231, \mathrm{~cm}^{-1} ;{ }^{1} \mathrm{H}$ NMR $\left(400 \mathrm{MHz}, \mathrm{CDCl}_{3}\right): \delta=15.25(\mathrm{~d}, \mathrm{~J}=11.2 \mathrm{~Hz}, 2 \mathrm{H}, \mathrm{H}-\mathrm{f}), 10.28(\mathrm{~d}, \mathrm{~J}=11.2 \mathrm{~Hz}, 2 \mathrm{H}$, H-h), 8.58 (s, 2H, H-b), 8.28 (s, 2H, H-e), 7.54 (s, 2H, H-g), $7.30-7.60$ (m, 8H, H-, j, k, l, m), 3.81 (m, 2H, $\mathrm{H}-\mathrm{d}$ ), 2.05 (s, 6H, H-a), 1.52 (d, $12 \mathrm{H}, \mathrm{H}-\mathrm{c}) ;{ }^{13} \mathrm{C} \mathrm{NMR}\left(100 \mathrm{MHz}, \mathrm{CDCl}_{3}\right): \delta=173.1,160.8,158.5,153.7$, $150.3,146.1,136.3,132.5,128.9,127.9,121.1,120.3,119.8,119.8,119.1,117.1,116.9,116.7,115.4,105.7$, 26.3, 20.1, 20.2 ppm; HRMS EI (m/z): calcd for $\mathrm{C}_{42} \mathrm{H}_{38} \mathrm{~F}_{2} \mathrm{~N}_{2} \mathrm{O}_{6}, 704.2695$; found, 704.2698.

8,8'- bis ( (E) -(3-fluorophenylimino) methyl) -5,5'-diisopropyl-3,3'-dimethyl-2,2' -binaphthyl-1,1',6,6',7,7' -hexaol (3b): yellow solid, Yield: 0.58 g, yield: $71 \%$; $\mathrm{mp}, 263^{\circ} \mathrm{C}-265^{\circ} \mathrm{C}$; IR $\left(\mathrm{KBr}, \mathrm{cm}^{-1}\right)$ : 3357, 3100, 1580, $1223, \mathrm{~cm}^{-1} ;{ }^{1} \mathrm{H}$ NMR $\left(400 \mathrm{MHz}, \mathrm{CDCl}_{3}\right): \delta=15.12(\mathrm{~d}, \mathrm{~J}=12.0 \mathrm{~Hz}, 2 \mathrm{H}, \mathrm{H}-\mathrm{f}), 10.20(\mathrm{~d}, \mathrm{~J}=12.0 \mathrm{~Hz}, 2 \mathrm{H}$, H-h), 8.57 (s, 2H, H-b), 8.27 (s, 2H, H-e), 7.56 (s, 2H, H-g), $7.31-7.62$ (m, 8H, H-i, k, l, m), 3.80 (m, 2H, $\mathrm{H}-\mathrm{d}$ ), 2.05 (s, 6H, H-a), 1.52 (d, $12 \mathrm{H}, \mathrm{H}-\mathrm{c}) ;{ }^{13} \mathrm{C}$ NMR $\left(100 \mathrm{MHz}, \mathrm{CDCl}_{3}\right.$ ): $\delta=173.0,160.8,158.5,153.6$, $150.1,146.2,136.3,132.5,128.9,127.9,121.3,120.3,119.1,119.0,118.9,117.1,116.9,116.2,115.2,105.6$, 26.2, 20.1, 20.1 ppm; HRMS EI (m/z): calcd for $\mathrm{C}_{42} \mathrm{H}_{38} \mathrm{~F}_{2} \mathrm{~N}_{2} \mathrm{O}_{6}, 704.2695$; found, 704.2698.

8,8' -bis ( (E) -(4-fluorophenylimino) methyl) -5,5' -diisopropyl-3,3' -dimethyl-2,2' - binaphthyl-1,1',6,6',7,7' -hexaol (3c): yellow solid, Yield: 0.69 g, yield: $85 \%$; mp, $265^{\circ} \mathrm{C}-267^{\circ} \mathrm{C}$; IR $\left(\mathrm{KBr}, \mathrm{cm}^{-1}\right)$ : 3351, 3188, 1580 , $1210, \mathrm{~cm}^{-1} ;{ }^{1} \mathrm{H}$ NMR $\left(400 \mathrm{MHz}, \mathrm{CDCl}_{3}\right): \delta=14.94(\mathrm{~d}, \mathrm{~J}=11.6 \mathrm{~Hz}, 2 \mathrm{H}, \mathrm{H}-\mathrm{f}), 10.31$ (d, J = 11.6 Hz, $2 \mathrm{H}$, H-h), 8.54 (s, 2H, H-b), 8.24 (s, 2H, H-e), 7.52 (s, 2H, H-g), $7.29-7.57$ (m, 8H, H-i, j, l, m), 3.80 (m, 2H, $\mathrm{H}-\mathrm{d}), 2.04(\mathrm{~s}, 6 \mathrm{H}, \mathrm{H}-\mathrm{a}), 1.51$ (d, $12 \mathrm{H}, \mathrm{H}-\mathrm{c}) ;{ }^{13} \mathrm{C}$ NMR $\left(100 \mathrm{MHz}, \mathrm{CDCl}_{3}\right): \delta=173.6,160.9,158.5,153.9$, $150.1,146.1,136.3,132.5,128.9,127.8,121.1,119.8,119.8,117.1,116.9,116.7,115.4,105.6,26.6,20.2,20.2$ ppm; HRMS EI (m/z): calcd for $\mathrm{C}_{42} \mathrm{H}_{38} \mathrm{~F}_{2} \mathrm{~N}_{2} \mathrm{O}_{6}, 704.2695$; found, 704.2698.

5,5' -diisopropyl-3,3' -dimethyl-8,8'-bis((E) -(2 -(trifluoromethyl) phenylimino) methyl)-2,2' -binaphthyl1,1',6,6',7,7'-hexaol (3d): yellow solid, Yield: $0.53 \mathrm{~g}$, yield: $64 \%$; $\mathrm{mp}, 251^{\circ} \mathrm{C}-253^{\circ} \mathrm{C}$; IR $\left(\mathrm{KBr}, \mathrm{cm}^{-1}\right): 3360$, $3120,1620,1330, \mathrm{~cm}^{-1} ;{ }^{1} \mathrm{H}$ NMR $\left(400 \mathrm{MHz}, \mathrm{CDCl}_{3}\right): \delta=15.21(\mathrm{~d}, \mathrm{~J}=11.0 \mathrm{~Hz}, 2 \mathrm{H}, \mathrm{H}-\mathrm{f}), 10.10(\mathrm{~d}, \mathrm{~J}=11.0$ $\mathrm{Hz}, 2 \mathrm{H}, \mathrm{H}-\mathrm{h}), 7.74$ (s, 2H, H-b), 7.62 (s, 2H, H-e), 7.68 - 7.26 (m, 8H, H-j, k, l, m), 5.77 (s, 2H, H-g), 3.76 $3.66(\mathrm{~m}, 2 \mathrm{H}, \mathrm{H}-\mathrm{d}), 2.15(\mathrm{~s}, 6 \mathrm{H}, \mathrm{H}-\mathrm{a}), 1.57-1.51(\mathrm{~m}, 12 \mathrm{H}, \mathrm{H}-\mathrm{c}) ; 13 \mathrm{C}$ NMR (100 MHz, $\left.\mathrm{CDCl}_{3}\right), \delta 175.0$, $154.8,149.5,146.8,138.6,133.5,133.3,130.2,129.5,127.1,127.1,127.0,126.9,125.3,125.0,122.3,121.2$, $120.9,119.0,118.9,116.4,114.2,106.4,27.6,20.2,20.2,20.1 \mathrm{ppm}$; HRMS EI (m/z): calcd for $\mathrm{C}_{44} \mathrm{H}_{38} \mathrm{~F}_{6} \mathrm{~N}_{2} \mathrm{O}_{6}$, 804.2630; found, 804.2634.

5,5'-diisopropyl-3,3'-dimethyl-8,8'-bis((E)-(3-(trifluoromethyl)phenylimino)methyl)-2, 2'-binaphthyl1,1',6,6',7,7'-hexaol (3e): yellow solid, Yield: $0.68 \mathrm{~g}$, yield: $\mathrm{n} 83 \%$; $\mathrm{mp}, 262^{\circ} \mathrm{C}-264^{\circ} \mathrm{C}$; $\mathrm{IR}\left(\mathrm{KBr}, \mathrm{cm}^{-1}\right): 3360$, $3120,1620,1328, \mathrm{~cm}^{-1} ;{ }^{1} \mathrm{H}$ NMR $\left(400 \mathrm{MHz}, \mathrm{CDCl}_{3}\right): \delta=15.02(\mathrm{~d}, \mathrm{~J}=11.7 \mathrm{~Hz}, 2 \mathrm{H}, \mathrm{H}-\mathrm{f}), 10.14$ (d, J = 11.7 $\mathrm{Hz}, 2 \mathrm{H}, \mathrm{H}-\mathrm{h}$ ), 7.79 (s, 2H, H-b), 7.65 (s, 2H, H-e), 7.54-7.41 (m, 8H, H-i, k, l, m), 5.79 (s, 2H, H-g), 3.77 $3.69(\mathrm{~m}, 2 \mathrm{H}, \mathrm{H}-\mathrm{d}), 2.16(\mathrm{~s}, 6 \mathrm{H}, \mathrm{H}-\mathrm{a}), 1.58-1.53(\mathrm{~m}, 12 \mathrm{H}, \mathrm{H}-\mathrm{c})$; 13C NMR (100 MHz, $\left.\mathrm{CDCl}_{3}\right), \delta 175.2$, $153.5,149.5,146.9,140.2,133.3,133.1,132.7,132.3,132.0,130.5,130.1,129.6,124.9,122.2,122.1,122.1$, $121.2,119.0,116.4,114.9,114.9,114.1,105.7,27.6,20.3,20.2,20.1 \mathrm{ppm}$; HRMS EI (m/z): calcd for $\mathrm{C}_{44} \mathrm{H}_{38} \mathrm{~F}_{6} \mathrm{~N}_{2} \mathrm{O}_{6}$, 804.2630; found, 804.2634.

5,5' -diisopropyl-3,3' -dimethyl-8,8'-bis((E)-(4-(trifluoromethyl) phenylimino) methyl) -2,2' -binaphthyl$1,1^{\prime}, 6,6^{\prime}, 7,7^{\prime}$-hexaol (3f): yellow solid, Yield: $0.51 \mathrm{~g}$, yield: $62 \%$; $\mathrm{mp}, 261^{\circ} \mathrm{C}-263^{\circ} \mathrm{C}$; IR $\left(\mathrm{KBr}, \mathrm{cm}^{-1}\right): 3365$, $3129,1622,1335, \mathrm{~cm}^{-1} ;{ }^{1} \mathrm{H}$ NMR $\left(400 \mathrm{MHz}, \mathrm{CDCl}_{3}\right): \delta=14.89(\mathrm{~d}, \mathrm{~J}=12.0 \mathrm{~Hz}, 2 \mathrm{H}, \mathrm{H}-\mathrm{f}), 10.15$ (d, J $=12.0$ $\mathrm{Hz}, 2 \mathrm{H}, \mathrm{H}-\mathrm{h}$ ), 7.77 (s, 2H, H-b), $7.65-7.55$ (m, 6H, H-e, i, j, l, m), 7.37 (d, J = 8.4 Hz, 4H), 5.75 (s, 2H, $\mathrm{H}-\mathrm{g}), 3.78$ - 3.68 (m, 2H, H-d), 2.16 (s, 6H, H-a), 1.58 - 1.55 (m, 12H, H-c); 13C NMR (100 MHz, $\mathrm{CDCl}_{3}$ ), ${ }^{13} \mathrm{C}$ NMR $\left(100 \mathrm{MHz}, \mathrm{CDCl}_{3}\right): \delta=175.5,153.1,149.8,149.6,146.9,142.4,140.9,133.4,130.2,129.7,127.2$, 127.1, 127.1, 119.0, 117.9, 116.5, 105.8, 105.8, 27.6, 20.3, 20.2, 20.1; HRMS EI (m/z): calcd for $\mathrm{C}_{44} \mathrm{H}_{38} \mathrm{~F}_{6} \mathrm{~N}_{2} \mathrm{O}_{6}$, 804.2630; found, 804.2634.

\subsection{Anti-Tumor Activities}

All the fluoride-containing gossypol derivatives were screened for in vitro cytotoxicity against three human cancer cell lines (HeLa, A-549 and BGC-823) and a normal cell line (VEC) by MTT assay. In vitro, the cytotoxic activities of gossypol and fluoride-containing gossypol Schiff base derivatives were deter- 
mined by the MTT cytotoxicity assay, which was performed in 96-well plates. The tumor cell line panel consisted of HeLa (human cervical carcinoma), A-549 (human lung carcinoma), BGC-823 (human gastric carcinoma), VEC (human vascular endothelial cells) (final concentration in the growth medium was $(2$ 4) $\left.\times 10^{4} / \mathrm{mL}\right)$. MTT solution $(20 \mu \mathrm{L} /$ well $)$ was added after cells were treated with drug for $48 \mathrm{~h}$, and cells were incubated for a further $4 \mathrm{~h}$ at $37^{\circ} \mathrm{C}$. The purple form azan crystals were dissolved in $150 \mu \mathrm{L}$ DMSO. After $5 \mathrm{~min}$, the plates were read on an automated micro plate spectrophotometer at $570 \mathrm{~nm}$. Assays were performed in triplicate in three independent experiments. The concentration required for $50 \%$ inhibition of cell viability $\left(\mathrm{IC}_{50}\right)$ what was calculated. In all of these experiments, three replicate wells were used to determine each point.

As shown in Table 2, it was found that substituent changes on the gossypol's aldehyde groups have a great influence on the normal cells activity $(\mathbf{3 a}-\mathbf{3 f})$, which exhibited lessened cytotoxicity against normal cells (VEC). The data reveal that compounds $3 \mathrm{a}, 3 \mathrm{c}$ and $3 \mathrm{f}$ exhibited superior anticancer activity against HeLa, compounds $3 b, 3 c, 3 e$ and $3 f$ exhibited superior anticancer activity against A-549, compounds $3 b$, $3 \mathrm{c}$ and $3 \mathrm{f}$ exhibited superior anticancer activity against BGC-823 compared to gossypol. In particular, fluorine substituent at the para positions of the phenyl ring showed remarkable inhibitory effects on HeLa (3c: $\mathrm{IC}_{50}=14.2 \mu \mathrm{M}, 3 \mathrm{f}: \mathrm{IC}_{50}=8.34 \mu \mathrm{M}$ ), A-549 (3c: $\mathrm{IC}_{50}=6.32 \mu \mathrm{M}, 3 \mathrm{f}: \mathrm{IC}_{50}=9.76 \mu \mathrm{M}$ ) and BGC-823 cells $\left(3 \mathrm{c}: \mathrm{IC}_{50}=8.62 \mu \mathrm{M}, 3 \mathrm{f}: \mathrm{IC}_{50}=4.36 \mu \mathrm{M}\right)$, which represented superior antitumor activity compared to gossypol $\left(\mathrm{IC}_{50}=23.3 \mu \mathrm{M}\right.$ against $\mathrm{HeLa}, \mathrm{IC}_{50}=19.1 \mu \mathrm{M}$ against $\mathrm{A}-549, \mathrm{IC}_{50}=17.1 \mu \mathrm{M}$ against BGC-823, $)$, respectively. Moreover, fluoride-containing gossypol derivatives $3 \mathrm{c}$ and $3 \mathrm{f}$ exhibit good safety profiles $\left(\mathrm{IC}_{50}>100 \mu \mathrm{M}\right.$ against VEC).

\section{CONCLUSION}

In summary, a series of novel fluoride-containing gossypol Schiff base derivatives were synthesized and tested for their in vitro cytotoxic activities against three human cancer cell lines(HeLa, A-549 and BGC-823) and a normal cell line (VEC) by using MTT cell proliferation assays. All the compounds exhibited lessened cytotoxicity against normal cells (VEC). Results revealed that compounds $\mathbf{3 a}, \mathbf{3 c}$ and $\mathbf{3 f}$ exhibited superior anticancer activity against HeLa, compounds $\mathbf{3 b}, \mathbf{3 c}, \mathbf{3 e}$ and $\mathbf{3 f}$ exhibited superior anticancer activity against A-549, compounds $3 \mathrm{~b}, 3 \mathrm{c}$ and $3 \mathrm{f}$ exhibited superior anticancer activity against BGC-823 compared to gossypol. In particular, fluorine substituent at the para positions of the phenyl ring showed remarkable inhibitory effects on HeLa (3c: IC $\left.\mathrm{IC}_{50}=14.2 \mu \mathrm{M}, 3 \mathrm{f}: \mathrm{IC}_{50}=8.34 \mu \mathrm{M}\right)$, A-549 (3c: $\mathrm{IC}_{50}=$ $\left.6.32 \mu \mathrm{M}, 3 \mathrm{f}: \mathrm{IC}_{50}=9.76 \mu \mathrm{M}\right)$ and BGC-823 cells $\left(3 \mathrm{c}: \mathrm{IC}_{50}=8.62 \mu \mathrm{M}, 3 \mathrm{f}: \mathrm{IC}_{50}=4.36 \mu \mathrm{M}\right)$. Moreover, fluoride-containing gossypol derivatives $3 \mathrm{c}$ and $3 \mathrm{f}$ exhibit good safety profiles $\left(\mathrm{IC}_{50}>100 \mu \mathrm{M}\right.$ against VEC). And thus as anti-cancer drug, fluoride-containing gossypol Schiff base derivatives has a better application prospects. Studies to determine the in vivo pharmacokinetics and efficacy of compounds $3 \mathrm{c}$ and $3 \mathrm{f}$ against some selected tumor cell lines are currently underway.

Table 2. The inhibiting effect of compounds $3 \mathrm{a}-3 \mathrm{f}$ to HeLa, A-549 and BGC-823 cell lines in vitro.

\begin{tabular}{ccccc}
\hline \multirow{2}{*}{ Compd. } & Normal cells IC $50 / \mu \mathrm{Mol} \cdot \mathrm{L}^{-1}$ & \multicolumn{3}{c}{ Cancer cells $\mathrm{IC}_{50} / \mu \mathrm{Mol} \cdot \mathrm{L}^{-1}$} \\
\cline { 2 - 5 } & VEC & HeLa & A-549 & BGC-823 \\
\hline 3a & 83.4 & 19.6 & 20.7 & 19.1 \\
3b & 75.2 & 29.1 & 15.5 & 11.4 \\
3c & $>100$ & 14.2 & 6.32 & 8.62 \\
3d & 89.8 & 33.7 & 22.3 & 22.2 \\
3e & 98.4 & 23.6 & 12.7 & 18.9 \\
3f & $>100$ & 8.34 & 9.76 & 4.36 \\
gossypol & 36.5 & 23.3 & 19.1 & 17.1 \\
\hline
\end{tabular}

${ }^{\mathrm{a}} \mathrm{IC}_{50}$ values were the means of three independent experiments run in duplicate. 


\section{ACKNOWLEDGEMENTS}

The authors thank the High-level Talent Introduction Foundation of Southern Medical University (C1033520), Natural Science Foundation of China (81102823), Guangdong Natural Science Foundation (2014A030310342) and the Science and Technology Program of Guangdong Province (2015A010105015) for financial support.

\section{REFERENCES}

1. Zhang, Y.S., Yuan, J., Fang, Z.Z., et al. (2012) Gossypol Exhibits a Strong Influence towards UDP-Glucuronosyltransferase (UGT) 1A1, 1A9 and 2B7-Mediated Metabolism of Xenobiotics and Endogenous Substances. Molecules, 17, 4896-4903. https://doi.org/10.3390/molecules17054896

2. Tso, W.W. (1984) Gossypol Inhibits Ehrlich Ascites Tumor Cell Proliferation. Cancer Letters, 24, 257-261. https://doi.org/10.1016/0304-3835(84)90021-1

3. Wu, Y.W., Chik, C.L. and Knazek, R.A. (1989) An in vitro and in vivo Study of Antitumor Effects of Gossypol on Human SW-13 Adrenocortical Carcinoma. Cancer Research, 49, 3754-3758.

4. Gilbert, N.E., O'Reilly, J.E., Chang, C.J., Lin, Y.C. and Brueggemeier, R.W. (1995) Antiproliferative Activity of Gossypol and Gossypolone on Human Breast Cancer Cells. Life Sciences, 57, 61-67. https://doi.org/10.1016/0024-3205(95)00243-Y

5. Jaroszewski, J.W., Kaplan, O. and Cohen, J.S. (1990) Action of Gossypol and Rhodamine 123 on Wild Type and Multidrug-Resistant MCF-7 Human Breast Cancer Cells: 31P Nuclear Magnetic Resonance and Toxicity Studies. Cancer Research, 50, 6936-6943.

6. Wang, X., Wang, J., Wong, S.C., Chow, L.S., Nicholls, J.M., Wong, Y.C., Liu, Y., Kwong, D.L., Sham, J.S. and Tsa, S.W. (2000) Cytotoxic Effect of Gossypol on Colon Carcinoma Cells. Life Sciences, 67, 2663-2671. https://doi.org/10.1016/S0024-3205(00)00857-2

7. Mohammad, R.M., Wang, S., Banerjee, S., Wu, X., Chen, J. and Sarkar, F.H. (2005) Nonpeptidic Small-Molecule Inhibitor of Bcl-2 and Bcl-XL, (-)-Gossypol, Enhances Biological Effect of Genistein against BxPC-3 Human Pancreatic Cancer Cell Line. Pancreas, 31, 317-324. https://doi.org/10.1097/01.mpa.0000179731.46210.01

8. Huang, Y.W., Wang, L.S., Chang, H.L., Ye, W., Dowd, M.K., Wan, P.J. and Lin, Y.C. (2006) Molecular Mechanisms of(-)-Gossypol-Induced Apoptosis in Human Prostate Cancer Cells. Anticancer Research, 26, 1925-1933.

9. Bauer, J.A., Trask, D.K., Kumar, B., Los, G., Castro, J., Lee, J.S., Chen, J., Wang, S., Bradford, C.R. and Carey, T.E. (2005) Reversal of Cisplatin Resistance with a BH3 Mimetic, (-)-Gossypol, in Head and Neck Cancer Cells: Role of Wild-Type p53 and Bcl-xL. Molecular Cancer Therapeutics, 4, 1096-1104. https://doi.org/10.1158/1535-7163.MCT-05-0081

10. Wolter, K.G., Wang, S.J., Henson, B.S., Wang, S., Griffith, K.A., Kumar, B., Chen, J., Carey, T.E., Bradford, C.R. and D'Silva, N.J. (2006) (-)-Gossypol Inhibits Growth and Promotes Apoptosis of Human Head and Neck Squamous Cell Carcinoma in vivo. Neoplasia, 8, 163-172. https://doi.org/10.1593/neo.05691

11. Li, L., Liu, Y. and Wang, Q. (2015) Recent Progress in Gossypol Derivatives and Their Structure Activity Relationship. China Sciencepaper, 10, 1351-1357.

12. Vander Jagt, D.L., Deck, L.M. and Royer, R.E. (2000) Gossypol: Prototype of Inhibitors Targeted to Dinucleotide Folds. Current Medicinal Chemistry, 7, 479-498. https://doi.org/10.2174/09298667003375119

13. Montamat, E.E., Burgos, C., Gerez de Burgos, N.M., Rovai, L.E., Blanco, A. and Segura, E.L. (1982) Inhibitory Action of Gossypol on Enzymes and Growth of Trypanosoma cruzi. Science, 218, 288-289. https://doi.org/10.1126/science.6750791

14. Royer, R.E., Deck, L.M., Campos, N.M., Hunsaker, L.A. and Vander Jagt, D.L. (1986) Biologically Active Derivatives of Gossypol: Synthesis and Antimalarial Activities of Peri-Acylated Gossylic Nitriles. Journal of Medicinal 
Chemistry, 29, 1799-1801. https://doi.org/10.1021/jm00159a043

15. Gomez, M.S., Piper, R.C., Hunsaker, L.A., Royer, R.E., Deck, L.M., Makler, M.T. and Vander Jagt, D.L. (1997) Substrate and Cofactor Specificity and Selective Inhibition of Lactate Dehydrogenase from the Malarial Parasite P. falciparum. Molecular and Biochemical Parasitology, 90, 235-246.

16. Deck, L.M., Royer, R.E., Chamblee, B.B., Hernandez, V.M., Malone, R.R., Torres, J.E., Hunsaker, L.A., Piper, R.C., Makler, M.T. and Vander Jagt, D.L. (1998) Selective Inhibitors of Human Lactate Dehydrogenases and Lactate Dehydrogenase from the Malarial Parasite Plasmodium falciparum. Journal of Medicinal Chemistry, 41, 3879-3887. https://doi.org/10.1021/jm980334n

17. Yang, J., Zhang, F., Li, J., Chen, G., Wu, S., Ouyang, W., Pan, W., Yu, R., Yang, J. and Tien, P. (2012) Synthesis and Antiviral Activities of Novel Gossypol Derivatives. Bioorganic \& Medicinal Chemistry Letters, 22, 1415-1420.

18. Yang, J., Chen, G., Li, L.L., Pan, W., Zhang, F., Yang, J., Wu, S. and Tien, P. (2013) Synthesis and Antiviral Activities of Novel Gossypol Derivatives. Bioorganic \& Medicinal Chemistry Letters, 23, 2619-2623.

19. Yang, J., Li, J.-R., Yang, J.-X., Li, L.-L., Ouyang, W.-J., Wub, S.-W. and Zhang, F. (2014) Synthesis and Anti-HIV-1 Activity of the Conjugates of Gossypol with Oligopeptides and D-Glucosamine. Chinese Chemical Letters, 25, 1052-1056.

20. Shelley, M.D., Hartley, L., Groundwater, P.W. and Fish, R.G. (2000) Structure-Activity Studies on Gossypol in Tumor Cell Lines. Anticancer Drugs, 11, 209-216. https://doi.org/10.1097/00001813-200003000-00009

21. Zhang, L., Jiang, H., Cao, X., Zhao, H., Wang, F., Cui, Y. and Jiang, B. (2009) Chiral Gossypol Derivatives: Evaluation of Their Anticancer Activity and Molecular Modeling. European Journal of Medicinal Chemistry, 44, 3961-3972.

22. Niu, X., Li, S., Wei, F., Huang, J., Wu, G., Xu, L., Xu, D. and Wang, S. (2014) Apogossypolone Induces Autophagy and Apoptosis in Breast Cancer MCF-7 Cells in Vitro and in Vivo. Breast Cancer, 21, 223-230.

https://doi.org/10.1007/s12282-012-0372-Z

23. Zhan, W., Hu, X., Yi, J., An, Q. and Huang, X. (2015) Inhibitory Activity of Apogossypol in Human Prostate Cancer in Vitro and in Vivo. Molecular Medicine Reports, 11, 4142-4148.

https://doi.org/10.3892/mmr.2015.3326

24. Royer, R.E., Deck, L.M., Vander Jagt, T.J., Martinez, F.J., Mills, R.G., Young, S.A. and Vander Jagt, D.L. (1995) Synthesis and Anti-HIV Activity of 1,1'-Dideoxygossypol and Related Compounds. Journal of Medicinal Chemistry, 38, 2427-2432. https://doi.org/10.1021/jm00013a018

25. Hagmann, W.K. (2008) The Many Roles for Fluorine in Medicinal Chemistry. Journal of Medicinal Chemistry, 51, 4359-4369. https://doi.org/10.1021/jm800219f

26. Zhou, Y., Wang, J., Gu, Z., Wang, S., Zhu, W., Acena, J.L., Soloshonok, V.A., Izawa, K. and Liu, H. (2016) Next Generation of Fluorine-Containing Pharmaceuticals, Compounds Currently in Phase II-III Clinical Trials of Major Pharmaceutical Companies: New Structural Trends and Therapeutic Areas. Chemical Reviews, 116, 422-518. https://doi.org/10.1021/acs.chemrev.5b00392

27. Zhang, Q., Hu, Z., Shen, Q., Chen, Y. and Lu, W. (2017) Design, Synthesis and Anti-Proliferative Activities of 2,6-Substituted Thieno[3,2-d]pyrimidine Derivatives Containing Electrophilic Warheads. Molecules, 22, 788. https://doi.org/10.3390/molecules22050788

28. Muller, K., Faeh, C. and Diederich, F. (2007) Fluorine in Pharmaceuticals: Looking Beyond Intuition. Science, 317, 1881-1886. https://doi.org/10.1126/science.1131943 
Submit or recommend next manuscript to SCIRP and we will provide best service for you:

Accepting pre-submission inquiries through Email, Facebook, LinkedIn, Twitter, etc.

A wide selection of journals (inclusive of 9 subjects, more than 200 journals)

Providing 24-hour high-quality service

User-friendly online submission system

Fair and swift peer-review system

Efficient typesetting and proofreading procedure

Display of the result of downloads and visits, as well as the number of cited articles

Maximum dissemination of your research work

Submit your manuscript at: http://papersubmission.scirp.org/

Or contact ns@scirp.org 